\title{
The difference between medicine and magic is that magicians know what they are doing
}

\author{
Rodney J. Hicks
}

Published online: 16 October 2014

(C) Springer-Verlag Berlin Heidelberg 2014

When the German scientist Paul Ehrlich conceptualised the perfect therapeutic agent or "magic bullet" (magische Kugel), he foresaw therapies that would lead to death of the targeted organism without damaging the host. Modern medicine continues to be beguiled by the prospect of highly effective targeted therapies that are without toxicity. Great advances have recently been made in pinpointing potential therapeutic targets in cancer cells [1]. These include characterisation of specific oncogenic proteins critical to the growth or survival of cancer cells and that are amenable to inhibition by small molecules. Another example is the identification of cell surface proteins overexpressed by cancer cells that can be targeted by monoclonal antibodies to invoke immunological killing. Dramatic responses in cancers that were previously unresponsive to a wide range of conventional therapies initially led to magical thinking that such targeted therapies would be a panacea for cancer. Unfortunately, while these approaches have undoubtedly changed the clinical landscape for many cancers that previously had a dismal prognosis, it has been increasingly recognised that tumour heterogeneity and adaptive evolution lead almost inevitably to the development of resistance to treatment. The experience of patients with advanced malignant melanoma being treated with inhibitors of oncogenic signalling associated with mutations in the BRAF oncogene having dramatic responses to treatment [2] but subsequently developing resistance through a range of genomic alterations [3] is an instructive example. As if by slight of hand, cancers continue to taunt us that they might be amenable to cure by virtue of their differences from normal cells.

\footnotetext{
R. J. Hicks ( $\square)$

Cancer Imaging, the Peter MacCallum Cancer Centre and Molecular Imaging and Targeted Therapeutics Laboratory, the Sir Peter MacCallum Department of Oncology, The University of Melbourne, St Andrew's Place East, East Melbourne, VIC 3002, Australia e-mail: rod.hicks@petermac.org
}

Radionuclide therapy is essentially a form of targeted therapy. Indeed, it could be argued that the use of radioactive iodine to treat thyroid cancer represents the prototypical example of a molecular targeted therapy. Leveraging the expression of the sodium-iodide symporter, it has been recognised since the 1940s that the ability of differentiated thyroid cancer cells to concentrate iodine enables ${ }^{131}$ I to have almost miraculous therapeutic effects on even widely metastatic disease [4]. However, it was also apparent very early in the evaluation of this therapy that despite the high specificity of radioiodine for functioning thyroid cells, there is the potential for toxicity, including acute leukaemia, related to "off-target" effects [5]. Thus, from the very dawn of radionuclide therapy, there has been a conscious effort to balance its therapeutic benefits with the risk of acute and long-term toxicity.

Over the past decade there has been growing enthusiasm for the therapeutic use of radiolabelled somatostatin analogues (SSAs) for the treatment of neuroendocrine tumours (NETs). For a disease in which responses to chemotherapy are modest both in number and degree, peptide receptor radionuclide therapy (PRRT) using either ${ }^{177} \mathrm{Lu}$-octreotate or ${ }^{90} \mathrm{Y}$ octreotide has shown impressive efficacy whether used alone [6-12] or combined with radiosensitising chemotherapy [13-16]. While most patients in these series had either symptomatic or progressive metastatic disease, PRRT has also been shown to cause regression of unresectable primary tumours [17]. These therapeutic effects have generally been associated with modest toxicity profiles. Evaluation of acute haematological toxicity has revealed relatively minor reductions in blood counts even in patients receiving concurrent radiosensitising chemotherapy $[14,18]$. Nephrotoxicity is a more serious potential long-term consequence of PRRT due to reabsorption of radiolabelled peptides by the proximal convoluted tubules. Renal toxicity has been shown to be more common when ${ }^{90} \mathrm{Y}$ is the therapeutic radionuclide $[9,19]$ than when ${ }^{177} \mathrm{Lu}$ is used [20,21], which is expected given that the 
longer beta particle path length of ${ }^{90} \mathrm{Y}$ than ${ }^{177} \mathrm{Lu}$ brings more glomeruli within range of particulate radiation arising from reabsorbed peptide. ${ }^{177} \mathrm{Lu}$-labelled SSAs, in particular, appear to have very low rates of long-term renal toxicity $[22,23]$. The routine use of amino acid infusions to inhibit tubular uptake [24] is thought to reduce the likelihood of nephrotoxicity, and the development of new ligands with higher affinity for tumour [25] will also likely also reduce the risk of radiationinduced renal impairment. However, conditions such as diabetes and hypertension, which are typically associated with decreased renal cortical thickness, have been recognised as risk factors for renal impairment following PRRT [21]. Against this excellent safety background, the occurrence of myelodysplastic syndrome (MDS) or acute leukaemia has been recognised as rare but serious events in patients receiving PRRT. The first reports of these complications were in association with high-administered activities of ${ }^{111}$ Indiethylenetriaminepentaacetic acid (DTPA)-octreotide delivered therapeutically to cumulative activities in excess of $100 \mathrm{GBq}$ [26] but have also been seen in patients receiving ${ }^{177} \mathrm{Lu}$ [8] and ${ }^{90} \mathrm{Y}$ SSAs [9].

In an article recently published in the European Journal of Nuclear Medicine and Molecular Imaging, Bodei et al. [27] analyse the toxicity associated with PRRT in 807 patients with NET treated at their facility. This series provides further evidence of the low side effect profile of PRRT. The majority of patients received ${ }^{90} \mathrm{Y}$-DOTA-octreotide either as their sole radiopeptide $(44 \%)$ or in combination with ${ }^{177}$ Lu-DOTAoctreotate $(19.5 \%)$. Most of the remaining patients (36\%) received ${ }^{177} \mathrm{Lu}$-DOTA-octreotate alone. As with many similar retrospective series in which treatment was largely being delivered on a compassionate basis during a period when knowledge and experience with PRRT was evolving, treatment regimens in individual patients appear to have been personalised and adapted based on both response and toxicity observed during treatment. While this approach can be justified on first principles [28], it is, however, unclear what criteria were used to choose either the radiopeptide or the administered activity. For example, it is not known whether ${ }^{90}$ Y-DOTA-octreotide was specifically avoided in patients with pre-existing renal impairment or risk factors for nephrotoxicity or whether there was a reduced administered activity in such patients compensating for the longer circulation time due to decreased renal clearance. Neither is it known whether tumour burden influenced the choice or administered activity of radiopeptide. Due to the "tumour sink" effect, it is theoretically possible to administer higher activities to patients with larger disease burdens without significantly increasing radiation dose to the kidneys [29].

Despite the limitations that are intrinsic in retrospective series, the authors provide a comprehensive summary of the toxicity profile of PRRT with a median follow-up of 30 months. Supporting previous data, often from smaller patient populations, ${ }^{90}$ Y-based PRRT regimens were associated with a higher incidence of persistent renal impairment than those receiving ${ }^{177}$ Lu-based PRRT. However, a cohort of patients treated before 1999, which did not receive renoprotective amino acid infusion, potentially confounds this observation. Nevertheless, persisting severe nephrotoxicity occurred in only $1.5 \%$ of the 807 patients. Similarly, although both MDS and acute leukaemia were observed, they were both uncommon and late complications. Overall, 19 $(2.35 \%)$ patients developed MDS at a mean of 1,351 days after the onset of PRRT. Of these, six subsequently went on to develop acute leukaemia while a further two patients presented with de novo leukaemia with the onset in these eight patients $(1.1 \%)$ occurring a mean of 1,699 days after onset of PRRT. Thus, overall, 21/807 (2.6\%) developed a secondary neoplastic marrow disorder potentially related to radiation exposure.

While these infrequent but severe complications might seem a low price to pay for the significant response rates and relatively long reported progression-free and overall survival reported in prior series, it behoves those who deliver PRRT to try to determine if there are any factors that influence the risk of acute and long-term complications. This admirable goal has been investigated in the current study by means of rather detailed and complex statistical analyses including modelling of clinical predictors associated with both nephrotoxicity and development of secondary haematological neoplasia. Quite appropriately, patient-related factors, including age at diagnosis, sex and prior treatments, or features relevant to tumour biology, including grade and proliferative fraction (Ki-67), were considered. Unfortunately, despite the authors' best efforts only a minority of the observed toxicities could be modelled by the identified clinical risk factors. This applied for both nephrotoxicity and development of MDS or acute leukaemia. Nevertheless, severe nephrotoxicity was shown to be significantly associated with the presence of hypertension and older age, both of which are known to be associated with progressive loss of glomerular function even in the absence of PRRT. Somewhat unexpectedly, however, neither MDS nor acute leukaemia was significantly associated with prior chemotherapy. If clinical risk factors other than age and the presence of hypertension were not able to identify the need to adapt therapy, the authors reasoned that features of the treatment regimen including the radionuclide type, cumulative activity or duration of treatment might also be associated with toxicity and therefore modifiable. Again modelling failed to support this hypothesis. Although, ${ }^{90}$ Y-based PRRT was associated with a higher rate of nephrotoxicity than ${ }^{177} \mathrm{Lu}$ and duration of PRRT exposure was associated with development of MDS, modelling was again not able to identify a particular risk profile.

The authors have recognised that an important factor lacking in their characterisation of these patients is the radiation 
dose delivered during the course of PRRT to tumour sites and concomitantly to the kidneys and bone marrow. They attempted to redress this by analysing toxicity in a small subgroup of 34 patients in whom dosimetry estimates were available. However, in this limited sample, although the single patient with severe nephrotoxicity had an estimated radiation dose above the recommended threshold for exposure, this was not the highest radiation exposure in the cohort. Furthermore, many other patients above the recommended threshold had no significant nephrotoxicity. Additionally, none of the three patients who developed MDS received above the recommended threshold of 2 Gy to bone marrow. Despite the failure of conventional dosimetry based on planar whole-body imaging to predict toxicity in the current paper, the results do hint at the importance of radiation delivery to non-target tissues to the development of toxicity. For example, the authors found that the development of reduced haemoglobin $(\mathrm{Hb})$ levels after PRRT were a co-dependent association of nephrotoxicity. Since erythropoietin production occurs close to the proximal convoluted tubules [30], it could be postulated that radiation dose to these cells is an important causal factor of both low $\mathrm{Hb}$ levels and loss of glomerular function. Similarly, they found that reduced platelet counts soon after treatment were associated with the subsequent development of MDS, raising the possibility that acute damage to bone marrow leads to an increased risk of later malignant marrow transformation.

So where do these new data leave us? Should we simply accept that toxicity from PRRT is simply a random game of chance? Perhaps it is reasonable and pragmatic to continue using fixed administered activities of radiolabelled SSAs independent of patient characteristics, co-morbidities, prior treatments, disease burden and tumour biology or to simply stop treating patients if they fail to respond or develop toxicity. Similarly, the choice of radionuclide could reasonably be chosen based on local availability, costs and regulations with no compelling evidence of a significant risk-benefit profile advantage of one radiolabelled SSA over another. Maybe it does not matter how this magical treatment works but only that it does. Intuitively and scientifically, these propositions are, however, unappealing. As cogently argued in a recent commentary in this very journal [31], "There is no bullet with properties so magical that it would not benefit from the application of science". Indeed, it seems inevitable that radiation dose at the cellular level will determine both response in sites of disease and toxicity to normal tissues. While we certainly need better predictive dosimetry to inform safe and effective administered activities of radionuclide therapies, measuring dose delivery following each cycle of PRRT is becoming feasible through quantitative single photon emission computed tomography (SPECT)/CT [32] of cumulative tissue activity of ${ }^{177} \mathrm{Lu}$ over time or by positron emission tomography (PET)/ $\mathrm{CT}$ imaging of pair production associated with ${ }^{90} \mathrm{Y}$ decay [33].
More routine application of these techniques will certainly advance our understanding of the benefits and risks of PRRT.

Bodei and colleagues allude to the potential importance of host sensitivity to radiation, which is perhaps related to inherited genetic characteristics. While investigation of genomic predictors of radiosensitivity will undoubtedly also be important to understanding how and when to use radiation as a therapeutic agent, we must ensure that we first have accurate information about radiation dose delivery to tumour and non-target tissues. Without the ability to understand and articulate the radiobiology underpinning both the excellent responses and the occasional severe toxicities associated with PRRT, it runs the risk of being viewed as a three-card trick. Like magicians, we need to know intimately the basis of the magic that we perform.

\section{References}

1. Hanahan D, Weinberg RA. Hallmarks of cancer: the next generation. Cell 2011;144:646-74. doi:10.1016/j.cell.2011.02.013.

2. McArthur GA, Puzanov I, Amaravadi R, Ribas A, Chapman P, Kim $\mathrm{KB}$, et al. Marked, homogeneous, and early [18F] fluorodeoxyglucose-positron emission tomography responses to vemurafenib in BRAF-mutant advanced melanoma. J Clin Oncol 2012;30:1628-34. doi:10.1200/JCO.2011.39.1938.

3. Holderfield M, Deuker MM, McCormick F, McMahon M. Targeting RAF kinases for cancer therapy: BRAF-mutated melanoma and beyond. Nat Rev Cancer 2014;14:455-67. doi:10.1038/nrc3760.

4. Seidlin SM, Rossman I. Radioiodine therapy of metastases from carcinoma of the thyroid; a 6 -year progress report. J Clin Endocrinol Metab 1949;9:1122-37. doi:10.1210/jcem-9-11-1122.

5. Seidlin SM, Siegal E, Yalow AA, Melamed S. Acute myeloid leukemia following prolonged iodine-131 therapy for metastatic thyroid carcinoma. Science 1956;123:800-1.

6. Kwekkeboom DJ, Mueller-Brand J, Paganelli G, Anthony LB, Pauwels S, Kvols LK, et al. Overview of results of peptide receptor radionuclide therapy with 3 radiolabeled somatostatin analogs. J Nucl Med 2005;46 Suppl 1:62S-6S.

7. Kwekkeboom DJ, Teunissen JJ, Bakker WH, Kooij PP, de Herder WW, Feelders RA. Radiolabeled somatostatin analog [177Lu-DOTA0,Tyr3] octreotate in patients with endocrine gastroenteropancreatic tumors. J Clin Oncol 2005;23:2754-62. doi: $10.1200 / J C O .2005 .08 .066$

8. Kwekkeboom DJ, de Herder WW, Kam BL, van Eijck CH, van Essen M, Kooij PP, et al. Treatment with the radiolabeled somatostatin analog [177 Lu-DOTA 0,Tyr3]octreotate: toxicity, efficacy, and survival. J Clin Oncol 2008;26:2124-30. doi:10.1200/JCO.2007.15. 2553.

9. Imhof A, Brunner P, Marincek N, Brief M, Schindler C, Rasch H, et al. Response, survival, and long-term toxicity after therapy with the radiolabeled somatostatin analogue [90Y-DOTA]-TOC in metastasized neuroendocrine cancers. J Clin Oncol 2011;29:2416-23. doi: 10.1200/JCO.2010.33.7873.

10. Bushnell Jr DL, O’Dorisio TM, O’Dorisio MS, Menda Y, Hicks RJ, Van Cutsem E, et al. 90Y-edotreotide for metastatic carcinoid refractory to octreotide. J Clin Oncol 2010;28:1652-9. doi:10.1200/JCO. 2009.22.8585.

11. Sabet A, Haslerud T, Pape UF, Sabet A, Ahmadzadehfar H, Grünwald F, et al. Outcome and toxicity of salvage therapy with 
$177 \mathrm{Lu}$-octreotate in patients with metastatic gastroenteropancreatic neuroendocrine tumours. Eur J Nucl Med Mol Imaging 2014;41: 205-10. doi:10.1007/s00259-013-2547-z.

12. Ezziddin S, Attassi M, Yong-Hing CJ, Ahmadzadehfar H, Willinek W, Grünwald F, et al. Predictors of long-term outcome in patients with well-differentiated gastroenteropancreatic neuroendocrine tumors after peptide receptor radionuclide therapy with $177 \mathrm{Lu}-$ octreotate. J Nucl Med 2014;55:183-90. doi:10.2967/jnumed.113. 125336.

13. Kong G, Johnston V, Ramdave S, Lau E, Rischin D, Hicks RJ. Highadministered activity In-111 octreotide therapy with concomitant radiosensitizing $5 \mathrm{FU}$ chemotherapy for treatment of neuroendocrine tumors: preliminary experience. Cancer Biother Radiopharm 2009;24:527-33. doi:10.1089/cbr.2009.0644.

14. Claringbold PG, Price RA, Turner JH. Phase I-II study of radiopeptide $177 \mathrm{Lu}$-octreotate in combination with capecitabine and temozolomide in advanced low-grade neuroendocrine tumors. Cancer Biother Radiopharm 2012;27:561-9. doi:10.1089/cbr.2012. 1276.

15. Kong G, Thompson M, Collins M, Herschtal A, Hofman MS, Johnston V, et al. Assessment of predictors of response and longterm survival of patients with neuroendocrine tumour treated with peptide receptor chemoradionuclide therapy (PRCRT). Eur J Nucl Med Mol Imaging 2014;41:1831-44. doi:10.1007/s00259-0142788-5.

16. Kashyap R, Hofman MS, Michael M, Kong G, Akhurst T, Eu P, et al. Favourable outcomes of $177 \mathrm{Lu}$-octreotate peptide receptor chemoradionuclide therapy in patients with FDG-avid neuroendocrine tumours. Eur J Nucl Med Mol Imaging 2014. doi:10.1007/ s00259-014-2906-4.

17. Barber TW, Hofman MS, Thomson BN, Hicks RJ. The potential for induction peptide receptor chemoradionuclide therapy to render inoperable pancreatic and duodenal neuroendocrine tumours resectable. Eur J Surg Oncol 2012;38:64-71. doi:10.1016/j.ejso.2011.08.129.

18. Hubble D, Kong G, Michael M, Johnson V, Ramdave S, Hicks RJ. $177 \mathrm{Lu}$-octreotate, alone or with radiosensitising chemotherapy, is safe in neuroendocrine tumour patients previously treated with high-activity 111In-octreotide. Eur J Nucl Med Mol Imaging 2010;37:1869-75. doi:10.1007/s00259-010-1483-4.

19. Cwikla JB, Sankowski A, Seklecka N, Buscombe JR, NasierowskaGuttmejer A, Jeziorski KG, et al. Efficacy of radionuclide treatment DOTATATE Y-90 in patients with progressive metastatic gastroenteropancreatic neuroendocrine carcinomas (GEP-NETs): a phase II study. Ann Oncol 2010;21:787-94. doi:10.1093/annonc/ mdp372.

20. Valkema R, Pauwels SA, Kvols LK, Kwekkeboom DJ, Jamar F, de Jong $\mathrm{M}$, et al. Long-term follow-up of renal function after peptide receptor radiation therapy with (90)Y-DOTA(0), Tyr(3)-octreotide and (177)Lu-DOTA(0), Tyr(3)-octreotate. J Nucl Med 2005;46(1): 83S-91S.

21. Bodei L, Cremonesi M, Ferrari M, Pacifici M, Grana CM, Bartolomei $\mathrm{M}$, et al. Long-term evaluation of renal toxicity after peptide receptor radionuclide therapy with 90Y-DOTATOC and 177Lu-DOTATATE: the role of associated risk factors. Eur J Nucl Med Mol Imaging 2008;35:1847-56. doi:10.1007/s00259-0080778-1.

22. Kashyap R, Jackson P, Hofman MS, Eu P, Beauregard JM, Zannino $\mathrm{D}$, et al. Rapid blood clearance and lack of long-term renal toxicity of 177Lu-DOTATATE enables shortening of renoprotective amino acid infusion. Eur J Nucl Med Mol Imaging 2013;40:1853-60. doi:10. 1007/s00259-013-2504-x.

23. Sabet A, Ezziddin K, Pape UF, Reichman K, Haslerud T, Ahmadzadehfar H, et al. Accurate assessment of long-term nephrotoxicity after peptide receptor radionuclide therapy with (177)Luoctreotate. Eur J Nucl Med Mol Imaging 2014;41:505-10. doi:10. 1007/s00259-013-2601-x.

24. de Jong M, Rolleman EJ, Bernard BF, Visser TJ, Bakker WH, Breeman WA, et al. Inhibition of renal uptake of indium-111DTPA-octreotide in vivo. J Nucl Med 1996;37:1388-92.

25. Wild D, Fani M, Fischer R, Del Pozzo L, Kaul F, Krebs S, et al. Comparison of somatostatin receptor agonist and antagonist for peptide receptor radionuclide therapy: a pilot study. J Nucl Med 2014;55:1248-52. doi:10.2967/jnumed.114.138834.

26. De Jong M, Valkema R, Jamar F, Kvols LK, Kwekkeboom DJ, Breeman WA, et al. Somatostatin receptor-targeted radionuclide therapy of tumors: preclinical and clinical findings. Semin Nucl Med 2002;32:133-40. doi:10.1053/snuc.2002.31027.

27. Bodei L, Kidd M, Paganelli G. Long-term tolerability of PRRT in 807 patients with neuroendocrine tumours: the value and limitations of clinical factors. Eur J Nucl Med Mol Imaging 2014. doi:10.1007/ s00259-014-2893-5.

28. Hofman MS, Hicks RJ. Peptide receptor radionuclide therapy for neuroendocrine tumours: standardized and randomized, or personalized? Eur J Nucl Med Mol Imaging 2014;41:211-3. doi:10.1007/ s00259-013-2621-6.

29. Beauregard JM, Hofman MS, Kong G, Hicks RJ. The tumour sink effect on the biodistribution of 68Ga-DOTA-octreotate: implications for peptide receptor radionuclide therapy. Eur J Nucl Med Mol Imaging 2012;39:50-6. doi:10.1007/s00259-011-1937-3.

30. Pan X, Suzuki N, Hirano I, Yamazaki S, Minegishi N, Yamamoto M. Isolation and characterization of renal erythropoietin-producing cells from genetically produced anemia mice. PLoS One 2011;6:e25839. doi:10.1371/journal.pone.0025839.

31. Flux GD, Bardiès M, Lassmann M. Biting the magic bullet: celebrating a decade of the EANM Dosimetry Committee. Eur J Nucl Med Mol Imaging 2014;41:1-3. doi:10.1007/s00259-013-2589-2.

32. Beauregard JM, Hofman MS, Pereira JM, Eu P, Hicks RJ. Quantitative (177)Lu SPECT (QSPECT) imaging using a commercially available SPECT/CT system. Cancer Imaging 2011;11:56-66. doi:10.1102/1470-7330.2011.0012.

33. Lhommel R, Goffette P, Van den Eynde M, Jamar F, Pauwels S, Bilbao JI, et al. Yttrium-90 TOF PET scan demonstrates highresolution biodistribution after liver SIRT. Eur J Nucl Med Mol Imaging 2009;36:1696. doi:10.1007/s00259-009-1210-1. 